\title{
Decreased serum level of IL-12 in the course of ischemia and reperfusion during abdominal aortic surgery
}

\author{
Monika Jedynak ${ }^{1}$, Barbara Mroczko ${ }^{2}$, Andrzej Siemiatkowski ${ }^{1}$, Marek Gacko ${ }^{3}$, \\ Maciej Szmitkowski ${ }^{2}$
}

\author{
${ }^{1}$ Department of Anaesthesiology and Intensive Therapy, Medical University of Bialystok, Poland \\ ${ }^{2}$ Department of Biochemical Diagnostics, Medical University of Bialystok, Poland \\ ${ }^{3}$ Department of Vascular Surgery and Transplantology, Medical University of Bialystok, Poland
}

\begin{abstract}
Ischemic-reperfusion injury (IRI) is defined as tissue damage, organ dysfunction or failure developed in the course of inflammatory response following ischemia and reperfusion (IR). Abdominal aortic aneurysm (AAA) repair required IR of distal parts of the body carries a risk of organ injury and postoperative mortality of between $4 \%$ and $12 \%$. The aim of this study was the evaluation of IL-12 serum level during AAA repair in relation to IR. Blood samples were taken before surgery (Preop), before aortic unclamping $\left(\right.$ Pre- $\left.X_{\text {off }}\right), 90$ min after unclamping (90 min- $\mathrm{X}_{\text {off }}$ ) and $24 \mathrm{~h}$ after surgery (Postop) from 37 AAA patients; and before surgery (Preop), at 90 min of surgery (90 min-surg), at 180 min of surgery (180 min-surg) and $24 \mathrm{~h}$ after operation (stop) from ten patients scheduled for elective surgery of lumbar discopathy (SC); and once from ten healthy controls. IL-12 was measured using the ELISA technique. Preoperative IL-12 was higher in AAA $(0.21 \mathrm{pg} / \mathrm{ml})$ and SC $(0.31 \mathrm{pg} / \mathrm{ml})$ patients than in controls $(0.05 \mathrm{pg} / \mathrm{ml})$. A significant decrease in IL-12 $(0.09 \mathrm{pg} / \mathrm{ml})$ was observed at $90 \mathrm{~min}-\mathrm{X}_{\text {off }}$ in comparison to the preoperative value in AAA but not in the SC group. $24 \mathrm{~h}$ after surgery, IL-12 levels were still low in the AAA group $(0.13 \mathrm{pg} / \mathrm{ml})$, and nonsignificantly surpassed the preoperative value in the SC group $(0.36 \mathrm{pg} / \mathrm{ml})$. We conclude that operative injury was associated with increased IL-12 levels, and IR with decreased IL-12 levels. Diminished IL-12 during AAA repair might be associated with a higher risk of postoperative complications, but this needs further evaluation. (Folia Histochemica et Cytobiologica 2011; Vol. 49, No. 3, pp. 465-471)
\end{abstract}

Key words: surgery, interleukin-12, ischemia, reperfusion, abdominal aortic aneurysm

\section{Introduction}

Ischemic-reperfusion injury (IRI) is defined as tissue damage, organ dysfunction and/or failure developed in the course of inflammatory response following ischemia and reperfusion (IR) [1, 2]. Many procedures such as cardiac, orthopedic, vascular and transplant surgery, require mandatory organ ischemia. During the abdominal aortic aneurysm (AAA) repair, intra-

\footnotetext{
Correspondence address: M. Jedynak,

Department of Anaesthesiology and Intensive Therapy,

Medical University, Bialystok;

M.C. Sklodowskiej Str. 24A, 15-276 Bialystok, Poland;

tel.: (+ 48 85) 74683 60, fax: (+ 48 85) 74687 49;

e-mail: jedynaka@umwb.edu.pl
}

operative cross-clamping and unclamping of the aorta results in ischemia and reperfusion of distal parts of the body with the risk of kidney, spinal cord, intestines and skeletal muscles injury [3].

However, although the mortality rate for open elective AAA operations has been reduced to between $4 \%$ and $12 \%$, it is still higher than for other surgical procedures [4]. This necessitates a search for factors that may influence the outcome. Activation of the inflammatory process and disturbances of the immune response as a result of ischemia and reperfusion, are considered to be the crucial mechanism for the higher complication and mortality rate in AAA patients.

The innate immune system is responsible for the initial response to stress factors like pathogens or tissue injury. In the course of IRI, the innate immune 
response is triggered by non-infectious stimuli, partly by signaling via Toll-like receptors (TLRs) $[1,2]$. Activation of TLRs initiates an inflammatory response, and activation and maturation of dendritic cells (DC) and T-helper cells (Th). T cells constitute one of the primary arms of the adaptive immune response, and play an important role in ischemic injury [2]. Experimental studies have suggested that altered Th1 cells response may ameliorate the injury, since it has been shown that interferon-gamma (INF- $\gamma$ ) producing Th1 cells are deleterious, while interleukin-4 (IL-4) producing Th2 cells are protective in IRI $[1,5]$. It appears that $\mathrm{T}$ regulatory cells play a crucial role in the recovery phase of IRI.

$\mathrm{T}$ cell response to different infectious and noninfectious stimuli is regulated by the IL-12 family of cytokines [6]. IL-12, known also as natural killer (NK) stimulating factor, is required for the induction of INF- $\gamma$ production, which is critical for the induction of Th1 cells. This multifunctional cytokine acts as the key regulator of cell-mediated immune response by promoting IFN- $\gamma$ production, proliferation and cytolytic activity of NK and T cells. The biologically active form of the cytokine is IL-12p70, a heterodimeric protein that consists of a p35 and a p40 subunit. IL-12 is mainly secreted by monocytes, macrophages and DC after stimulation with lipopolysaccharide (LPS), tumor necrosis factor $\alpha(\mathrm{TNF} \alpha)$ and IFN- $\gamma$ [6]. To prevent systemic inflammation, IL-12 production must be inhibited. It is hypothesized that IL-12p40 and IL-4 possess an inhibitory role in IL-12p70 production, but this needs further evaluation [7].

It is generally agreed that trauma, surgery and infection induce pro-inflammatory and anti-inflammatory responses that contribute to cytokine production and secretion followed by infiltration of inflammatory cells $[8,9]$. As far as IL-12 is concerned, increased synthesis and secretion have been shown in the course of infection and injury of trauma patients [10]. In an experimental model of septic peritonitis, surgery- or sepsis-induced IL-12 production was protective against a lethal outcome, and neutralization of this cytokine led to increased mortality in spite of antibiotic administration [11]. The authors suggest that the lack of IL-12 disturbs the organization and the limitation of tissue damage. In accordance with this hypothesis, diminished IL-12 synthesizing capability before an operation was found to be a good predictor of fatal postoperative sepsis in patients with underlying malignancy $[12,13]$.

However, there is experimental and clinical evidence that diminished production of IL-12 is associated with IRI and should act protectively. Its role during AAA surgery with relation to ischemia-reper- fusion has yet to be elucidated. In our previous preliminary study, we showed a significant decrease in IL-12 serum level at 90 min of reperfusion during identical clinical conditions [14]. Because the reason for the observed changes could not be precisely explained, we decided to enlarge the study group and to widen the research to include patients scheduled for other surgical procedures not associated with ischemia and reperfusion.

The aim of this study was to evaluate IL-12 serum levels during elective open AAA repair in comparison with surgical procedures with no IR. We also assessed the relationship between observed changes and ischemia and reperfusion.

\section{Material and methods}

The Medical University of Bialystok Ethical Committee on research on humans and animals has approved this study. Written informed consent was obtained from all patients.

Thirty seven consecutive patients hospitalized in the Department of Vascular Surgery and Transplantology of the Medical University of Bialystok with a diagnosis of infrarenal AAA, and scheduled for open elective surgery, were included in the study. Exclusion criteria included a positive history of malignant, rheumatic or immunologic diseases. The surgical control (SC) group consisted of ten patients hospitalized in the Department of Neurosurgery of the Medical University of Bialystok with a diagnosis of lumbar discopathy, and scheduled for elective surgery. Exclusion criteria were those listed before, plus negative aortic pathology revealed on abdominal ultrasonography during the past six months. The healthy control (HC) group comprised ten healthy volunteers, who were medical staff. Patient characteristics and intraoperative data are summarized in Table 1.

The day before surgery, each patient was qualified by an anesthesiologist and preoperative risk was estimated according to American Society of Anesthesiologist (ASA) and Goldmann Cardiac Risk scores. In both surgical groups, midazolam was used as premedication. Operations were performed under general anesthesia with ethomidate and cis-atracurium for induction, and with sevoflurane, oxygen/ /air mixture and opioids for maintenance. Additionally, in AAA patients, continuous epidural anesthesia was provided unless contraindications were present. Antibiotic prophylaxis consisted of $1.0 \mathrm{~g}$ of cefazolinum on induction, followed by two further postoperative doses. Patients underwent elective AAA repair with bifurcated graft placement with identical stents (Uni-Graft KD.V) supplied by B/Braun (Easculap AG \& CO KG, Germany).

Peripheral venous blood samples were taken before surgery (Preop), before aortic unclamping (Pre- $\left.\mathrm{X}_{\text {off }}\right), 90 \mathrm{~min}$ after unclamping (90 min- $\mathrm{X}_{\text {off }}$ ), and $24 \mathrm{~h}$ after surgery 
Table 1. Patient characteristics, preoperative and intraoperative data

\begin{tabular}{|c|c|c|c|}
\hline & AAA & SC & HC \\
\hline Number of patients & 37 & 10 & 10 \\
\hline Age (years) & $68(44-79)^{*}$ & $60.5(48-68)$ & $67(50-73)$ \\
\hline ASA score II, n (\%) & $14(38 \%)$ & $10(100 \%)$ & - \\
\hline ASA score III, n (\%) & $19(51 \%)$ & 0 & - \\
\hline ASA score IV, n (\%) & $4(11 \%)$ & 0 & - \\
\hline Goldmann score & $8(3-29)$ & 0 & - \\
\hline Duration of surgery [min] & $225(150-310)^{*}$ & $147.5(110-220)$ & - \\
\hline Duration of clamping [min] & $60(40-90)$ & - & - \\
\hline Duration of hospital stay (days) & $13(1-34)^{*}$ & $8(5-12)$ & - \\
\hline Blood transfusion [ml] & $800(0-3500)$ & 0 & - \\
\hline Concomitant diseases, $\mathrm{n}(\%)$ & - & - & - \\
\hline Atherosclerotic vascular disease & $34(92 \%)^{*}$ & $3(30 \%)$ & - \\
\hline Hypertension & $24(65 \%)^{*}$ & $2(20 \%)$ & - \\
\hline Coronary heart disease & $22(59 \%)^{*}$ & $2(20 \%)$ & - \\
\hline Chronic heart failure & $6(16) \%$ & 0 & - \\
\hline Diabetes & $6(16 \%)$ & $1(10 \%)$ & - \\
\hline Smoking & $29(78 \%)$ & $4(40 \%)$ & - \\
\hline Aneurysm diameter [mm] & $42(35-70)$ & - & - \\
\hline
\end{tabular}

AAA - abdominal aortic aneurysm; $\mathrm{SC}$ - surgical control; $\mathrm{HC}$ - healthy control; data is shown as either medians with ranges or number of patients (n) and percentage (\%); ${ }^{*} \mathrm{p}<0.05$ AAA vs. SC

(Postop) from the AAA group; samples were taken before surgery (Preop), at 90 min of surgery (90 min-surg), at 180 min of surgery (180 min-surg) and $24 \mathrm{~h}$ after operation (Postop) from the SC patients; and once from the HC group. The time point of 180 min-surg in the SC patients was chosen because it corresponded the best with the time point of 90 min- $X_{\text {off }}$ in the AAA group. Three-ml samples of blood were taken with minimal stasis from the antecubital vein into tubes for serum and centrifugated within $10 \mathrm{~min}$ at 3,000 $\mathrm{rpm}$. The serum was removed into two-ml micro tubes and stored at a temperature of $-70^{\circ} \mathrm{C}$ until measurement. Levels of IL-12 heterodimer were measured twice in each sample with an enzyme-linked immunosorbent assay (ELISA) Quantikine, high sensitive kit (R\&D Systems, Abingdon, UK), according to the manufacturer's instructions. The manufacturer claims a sensitivity less than $0.5 \mathrm{pg} / \mathrm{ml}$ and intra-assay variability of $4.1 \%$ at a IL-12 concentration of $18.6 \mathrm{pg} / \mathrm{ml}$. Other tests such as blood cell count, electrolyte, blood gases, creatinine, bilirubin, albumin and total protein levels, aminotransferase activity, and coagulation tests, were measured using standard laboratory methods before and after surgery.

Statistical analysis was performed using STATISTICA 5.0 for Windows. Since the data was not normally distributed, the results were presented as median and range. For comparison within and between the groups, non-parametric Wilcoxon and Mann-Whitney U tests were used. Fish- er's Exact test was used to assess the relationship between two dichotomous variables. To analyze correlations, Spearman rank correlation coefficient was calculated. A p $<0.05$ was considered as statistically significant.

\section{Results}

Thirty seven consecutive patients undergoing AAA repair, and ten patients scheduled for elective surgery of lumbar discopathy (SC), were included in the study (Table 1). Of the AAA patients, 13 developed complications. Local complications like minor wound infection or thrombosis of the prosthesis arm were observed in three of them. In the other ten cases, five developed pneumonia, two postoperative hemostatic disorders, and in three patients decompensation of chronic heart failure with multiple organ dysfunction syndrome (MODS) was observed. Three patients died: one because of MODS in the course of pneumonia, and two because of MODS in the course of heart failure. All SC patients recovered uneventfully.

\section{Serum IL-12 levels in AAA and SC groups}

In AAA patients, serum IL-12 levels were significantly higher before surgery $(\mathrm{p}=0.021)$, before aortic 
Table 2. Serum level of IL-12 (pg/ml) before operation (Preop); before unclamping of the aorta (Pre- $\mathrm{X}_{\text {off }}$ ); 90 min after unclamping (90 min- $\mathrm{X}_{\text {off }}$ ); and $24 \mathrm{~h}$ after surgery (Postop) in abdominal aortic aneurysm (AAA) patients. Serum level of IL-12 (pg/ml) before operation (Preop); at 90 min of surgery (90 min-surg); at $180 \mathrm{~min}$ of surgery (180 min-surg); and $24 \mathrm{~h}$ after surgery (Postop) in surgical control (SC) patients and in healthy controls (HC). Results are presented as median and range

\begin{tabular}{|l|c|c|c|c|}
\hline HC & 0,05 & & & \\
& $(0,01-0,94)$ & & & \\
\hline \multirow{2}{*}{ AAA } & Preop & Pre- $\mathrm{X}_{\text {off }}$ & $90 \mathrm{~min}-\mathrm{X}_{\text {off }}$ & $0.13^{*}+$ \\
& $0.21^{*}+$ & $0.22^{*}+$ & 0.09 & $(0.01-4.94)$ \\
\hline SC & $(0.01-3.46)$ & $(0.01-4.28)$ & $(0.01-1.27)$ & Postop \\
& Preop & 90 min-surg & 180 min-surg & $0.36^{*}$ \\
& $0.31^{*}$ & $0.36^{*}+$ & 0.23 & $(0.04-0.90)$ \\
\hline
\end{tabular}

${ }^{*} \mathrm{p}<0.05$ (patients vs. HC),$+\mathrm{p}<0.05$ (90 min- $\mathrm{X}_{\text {off }}$ and $180 \mathrm{~min}$-surg vs. other time points within the group)

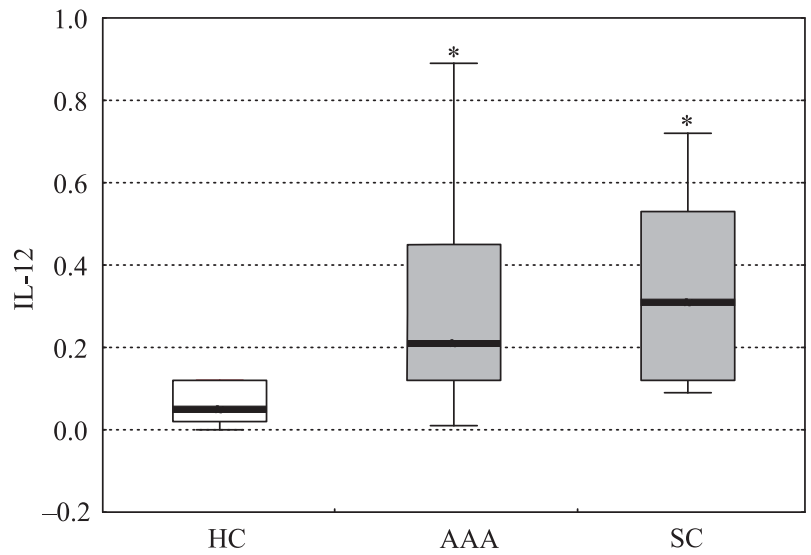

Figure 1. IL-12 serum levels $(\mathrm{pg} / \mathrm{ml})$ in abdominal aortic aneurysm (AAA) and surgical control (SC) groups before surgery in comparison to healthy controls (HC). Minimum, maximum, $25^{\text {th }}$ and $75^{\text {th }}$ percentiles (box) and median (line) values are showed. $* \mathrm{p}<0.05$ AAA and SC vs. HC

unclamping $(p=0.004)$ and $24 \mathrm{~h}$ after surgery $(p=0.04)$ than in the $\mathrm{HC}$ group (Table 2, Figure 1). We found a significant decrease in IL-12 levels at 90 minutes of reperfusion in comparison with the preoperative value $(\mathrm{p}<0.0001)$ (Figure 2). IL-12 levels at $90 \mathrm{~min}-\mathrm{X}_{\text {off }}$ were also lower than Pre- $\mathrm{X}_{\text {off }}(\mathrm{p}<0.0001)$ and postoperative $(\mathrm{p}=0.002)$ concentrations in the AAA group (Table 2).

Similarly, in SC patients, IL-12 concentrations were elevated before surgery $(\mathrm{p}=0.023)$, at $90 \mathrm{~min}$ of surgery $(p=0.015)$ and postoperatively $(p=0.04)$ compared to the HC group. We observed a slight, insignificant, decrease in cytokine levels at 180 min-surg in comparison with the preoperative value (Figure 2). Diminished IL-12 concentrations at 180 min of surgery were significantly lower compared to levels at 90 $\min -\operatorname{surg}(\mathrm{p}=0.008)$.

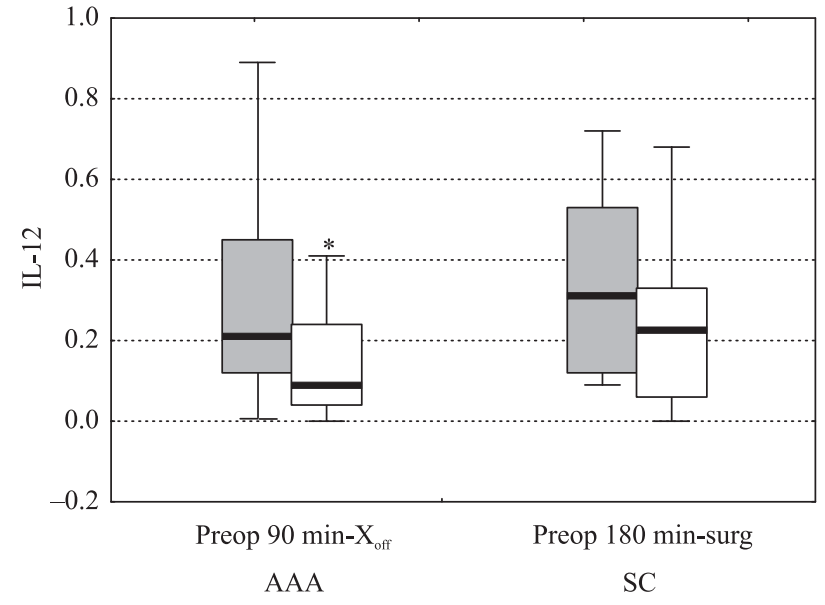

Figure 2. IL-12 serum levels (pg/ml) before surgery (Preop), at $90^{\text {th }}$ min of reperfusion $\left(90-\mathrm{min}-\mathrm{X}_{\text {off }}\right)$ and at $180^{\text {th }}$ min of surgery (180 min-surg) in abdominal aortic aneurysm (AAA) and surgical control (SC) groups. Minimum, maximum, $25^{\text {th }}$ and $75^{\text {th }}$ percentiles (box) and median (line) values are showed. ${ }^{*} \mathrm{p}<0.0590 \mathrm{~min} \mathrm{X}_{\text {off }}$ vs. Preop in AAA group

It is worth noting that $24 \mathrm{~h}$ after surgery, IL-12 levels reached, and even surpassed, the preoperative value in the SC group, unlike with AAA patients, whose postoperative cytokine levels remained low, and even lower than before the operation, although the observed differences were not statistically significant (Figure 3).

We found a lack of significant differences in IL-12 level between AAA and SC patients in every point of measurement, as well as between AAA patients with epidural anaesthesia and those without. There were also no significant differences detected in IL-12 levels between patients with and without complications or between those who died and those who recovered in the AAA group. 


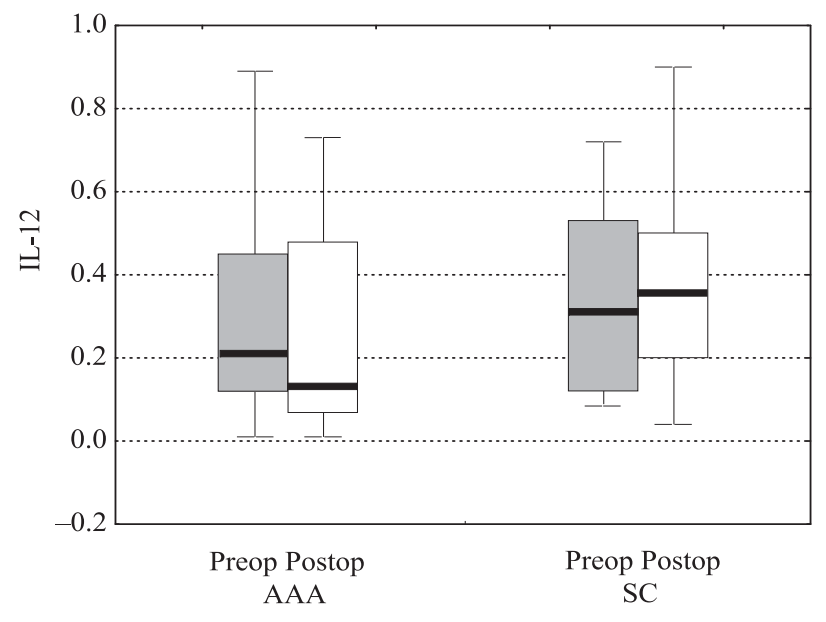

Figure 3. IL-12 serum levels (pg/ml) before surgery (Preop) and 24h after surgery (Postop) in abdominal aortic aneurysm (AAA) and surgical control (SC) groups. Minimum, maximum, $25^{\text {th }}$ and $75^{\text {th }}$ percentiles (box) and median (line) values are showed

\section{Correlation calculations}

All measured biochemical and hematological parameters were correlated with the levels of IL-12 in the AAA and SC groups, but there were no statistically significant relationships found. Nor were any significant correlations found between IL-12 levels in AAA patients and the postoperative clinical assessment according to Acute Physiology and Chronic Health Evaluation II score (APACHE II), Sequential Organ Failure Assessment (SOFA) or Multiple Organ Dysfunction score (MODs) (data not shown).

\section{Discussion}

As with every surgical procedure, AAA repair is associated with disturbances of innate and adaptive immune response, and the risk of local and systemic complications, including sepsis. Ischemia and reperfusion following clamping and declamping of the aorta additionally modifies the immune response generated already by an operative injury [3,9]. This leads to an enhanced complication risk in AAA patients compared to those undergoing other surgical procedures. IL-12 as an inductor of INF- $\gamma$ production from NK cells and a critical mediator of the type- 1 immune response is probably involved in the pathogenesis of organ dysfunction and high risk of death in the course of IRI. In our previous, preliminary, study we showed a significant decrease in IL-12 serum levels during IR in 17 patients undergoing AAA repair [14]. To make the data stronger, we decided to enlarge the group and to add a surgical control, which includes patients undergoing surgery not associated with IR.

We found significantly higher preoperative IL-12 serum levels in the AAA and SC groups compared to healthy controls. Elevated circulating levels of other cytokines such as IL- $1 \beta$, IL- 6 and TNF $\alpha$ in patients with AAA were reported by Juvonen et al. [15]. According to the authors, this response is indicative of chronic inflammation in AAA, and we suggest that the increased preoperative IL-12 may be a part of this response. However, we found no studies in the literature looking at IL-12 levels in AAA patients. It was also difficult to compare our results with IL-12 concentrations in other diseases, because different methods of measurement were used by various authors. Since SC patients also presented higher preoperative IL-12 concentrations compared to controls, the elevation of cytokine in both groups seems to result, at least in part, from perioperative stress. It is well established that the patient's immunological status before surgery is crucial for their postoperative immune response. High preoperative LPS-stimulated IL-12 production has been found to be associated with low risk of sepsis-related death in patients with malignant disease [12]. Similarly, patients who developed postoperative sepsis showed impaired preoperative production of IL-12 by human LPS-stimulated monocytes [13].

Consistently with a critical role for this cytokine for the host defense in sepsis, its neutralization led to impaired bacterial clearance, followed by markedly enhanced death rate in murine septic peritonitis [11]. In the light of these findings, we suppose that higher IL-12 preoperative levels resulted in part from perioperative stress and presented a protective mechanism against potential infective complications.

During the first two hours of surgery, i.e. at the end of ischemia in the AAA patients and after 90 minutes of surgery in the SC patients, IL-12 remained at similar levels as preoperatively in both groups, and they were still significantly higher than in the $\mathrm{HC}$ group. The enhanced production and secretion of IL-12 as a response to surgical injury has been well documented in 44 patients undergoing coronary artery bypass graft (CABG) surgery [8]. Those authors reported the elevation of IL-12 after institution of bypass in the on-pump group, and 30 min after induction of anesthesia in the off-pump group in comparison with the level measured just after the induction of anesthesia. This elevation persisted until skin closure and tended to be higher in the on-pump group. There was no healthy control group in this study with which to compare data. Wrigge et al. [16] also found elevated IL-12 concentrations after 1, 2, and 3 hours of abdominal, but not thoracic, surgery. They suggest- 
ed that this difference was related to the exposure to intestinal bacteria during abdominal surgery. Contrary to those, and to our, results, a lack of significant changes in IL-12 serum levels at the beginning and after surgery was found by Ahlers et al. [17] in 60 patients with cancer undergoing major abdominal surgery. In another study of 30 cancer patients, IL-12 levels were undetectable before operation and on the $1^{\text {st }}, 2^{\text {nd }}, 3^{\text {rd }}$ and $5^{\text {th }}$ days after surgery [18].

Such inconsistence between different studies may result from varying methods of cytokine measurement and the presence of neoplasmatic disease. The anti-tumor and antiangiogenic activities of IL-12 have been well documented in the literature, and different cytokine serum profiles can be expected in patients with malignant disease [19]. In this study, we used the high sensitivity ELISA method, which detects even small amounts of IL-12 in human plasma or serum, and we also excluded patients with a positive history of malignant, rheumatic or immunologic diseases. Our data confirmed that an operative injury is associated with elevated levels of IL-12 in the serum of patients.

In discussing variations of serum IL-12 levels, we should take into account the possible effect of medications, particularly anesthetics, that had been given to the patients before and during surgery. However Helmy et al. [9] and Wrigge et al. [16] showed that it was mainly the intensity of surgical injury, and not the anesthesia, that caused fluctuations in cytokines levels. We used a similar protocol in both surgery groups to minimize the effect of the anesthetic. There have only been a few reports on the influence of anesthesia on systemic IL-12 level, and no significant differences have been detected [17].

It is well established that ischemia and reperfusion disturb the pro-inflammatory and anti-inflammatory response. We observed a decrease in IL-12 level at 90 minutes of reperfusion in comparison with the preoperative value in AAA patients, with no corresponding significant changes in the SC group. Experimental data demonstrated that IL-12 promoted hepatic inflammation in IRI, and that blockade of endogenous cytokine significantly reduced liver accumulation of neutrophils and hepatocellular injury [20]. The protective role of diminished IL-12 production in experimental myocardial reperfusion injury was shown by Yang and Szabo [21]. The main endogenous factor affecting the level of IL-12 is IL-10. Significantly increased IL-12 level in IL-10-deficient mice was associated with significantly higher mortality during reperfusion compared to wild-type animals [21]. Similarly, a protective role of depleted IL-12 level has been found in kidney reperfusion injury [22]. On the basis of experimental data, we suggest that decreased
IL-12 level after IR in AAA patients might represent a protective mechanism against organ IRI. Significantly decreased IL-12 lung tissue level has been found during warm ischemia and reperfusion in the course of human lung transplantation procedure [23]. Contradicting our results, Castellheim et al. [8] noticed a significantly higher IL-12 level measured 60 minutes after institution of by-pass in on-pump CABG patients compared to the cytokine level at 90 min after induction of anesthesia in off-pump patients. Probably, the difference in the intensity of ischemia during AAA and CABG could explain such a distinction, but this has been not evaluated yet. Significantly lower LPS-stimulated monocyte IL-12 production has been observed in patients after 12 hours of treatment for hemorrhagic shock in comparison with controls [24].

Among clinical studies that assessed IL-12 level during ischemia and reperfusion, for the first time we have shown depleted cytokine level in the course of IR. The varying methods of measuring IL-12 in human fluids used by other authors, means that it is difficult to compare their data to ours.

We showed that IL-12 production during AAA repair was affected by at least two factors: an operative injury which was associated with a higher level of this cytokine, and IR which led to lower IL-12 production and secretion. As far as the data of other authors can be taken into account, we suggest that increased IL-12 level may be essential for a proper response to an operative injury and protection against postoperative complications. However, during this protective reaction, another protective mechanism was turned on to help guard against IR injury. This consists of ameliorating the activity of some cytokines including IL-12. In this condition, IL-12 protective features were not available, just as its unfavorable activity could not be inhibited. This is the situation when both protective mechanisms are ineffective because they are completely opposite. Thereby, immunological balance was quickly restored after surgery not associated with ischemia and reperfusion, but not in the AAA group. The median cytokine level in SC patients was even higher 24 hours after the operation than preoperatively, but it remained low in AAA patients. This might be one reason for the higher susceptibility of AAA patients to postoperative complications, since it has been shown that impaired production of IL-12 is associated with the risk of postoperative sepsis.

Summarizing our results, IL-12 levels increased during surgery. Ischemia and reperfusion during surgery resulted in a statistically significant decrease in 
IL-12, which persisted even 24 hours after surgery, although to a lesser degree. The decrease in IL-12 concentration during surgery differentiated the AAA patients from the SC patients. Taking into consideration the positive role of enhanced production of IL-12 during surgery as an important part of the response to operative injury, diminished IL-12 levels during AAA repair may be associated with a higher risk of postoperative complications in this group of patients. However, this needs further studies.

\section{Acknowledgements}

This work was supported by a research grant from the Medical University of Bialystok, Poland, no. 3-14559L.

\section{References}

1. Boros P, Bromberg JS. New cellular and molecular immune pathways in ischemia/reperfusion injury. Am $J$ Transplant. 2006;6:652-658.

2. Linfert D, Chowdhry T, Rabb H. Lymphocytes and ischemiareperfusion injury. Transplant Rev (Orlando). 2009;23:1-10.

3. Gelman S. The pathophysiology of aortic cross-clamping and unclamping. Anesthesiology. 1995;82:1026-1060.

4. Hallin A, Bergqvist D, Holmberg L. Literature review of surgical management of abdominal aortic aneurysm. Eur $J$ Vasc Endovasc Surg. 2001;22:197-204.

5. Ysebaert DK, De Greef KE, De Beuf A et al. T cells as mediators in renal ischemia/reperfusion injury. Kidney Int. 2004;66:491-496.

6. Lyakh L, Trinchieri G, Provezza L, Carra G, Gerosa F. Regulation of interleukin-12/interleukin-23 production and the T-helper 17 response in humans. Immunol Rev. 2008;226: 112-131.

7. Zhang S, Wang Q. Factors determining the formation and release of bioactive IL-12: regulatory mechanisms for IL-12p70 synthesis and inhibition. Biochem Biophys Res Commun. 2008;372:509-512.

8. Castellheim A, Hoel TN, Videm V et al. Biomarker profile in off-pump and on-pump coronary artery bypass grafting surgery in low-risk patients. Ann Thorac Surg. 2008;85:1994-2002.

9. Helmy SA, Wahby MA, El-Nawaway M. The effect of anaesthesia and surgery on plasma cytokine production. Anaesthesia. 1999;54:733-738.

10. Heizmann O, Koeller M, Muhr G, Oertli D, Schinkel C. Th1- and Th2-type cytokines in plasma after major trauma. J Trauma. 2008;65:1374-1378.
11. Steinhauser ML, Hogaboam CM, Lukacs NW, Strieter RM, Kunkel SL. Multiple roles for IL-12 in a model of acute septic peritonitis. J Immunol. 1999;162:5437-5443.

12. Novotny AR, Emmanuel K, Ulm K et al. Blood interleukin 12 as preoperative predictor of fatal postoperative sepsis after neoadjuvant radiochemotherapy. Br J Surg. 2006;93: $1283-1289$.

13. Weighardt H, Heidecke CD, Westerholt A, Emmanuilidis $\mathrm{K}$ et al. Impaired monocyte IL-12 production before surgery as a predictive factor for the lethal outcome of postoperative sepsis. Ann Surg. 2002;235:560-567.

14. Jedynak M, Siemiątkowski A, Gacko M, Mroczko B, Borkowski J, Szmitkowski M. Serum concentrations of interleukin-12 (IL-12) in patients undergoing abdominal aortic aneurysm repair - preliminary report. Pol Arch Med Wew. 2004;4:1173-1179.

15. Juvonen J, Surcel HM, Satta J et al. Elevated circulating levels of inflammatory cytokines in patients with abdominal aortic aneurysm. Arteriosclerosis, Thrombosis, and Vascular Biology. 1997;17:2843-2847.

16. Wrigge $\mathrm{H}$, Uhlig $\mathrm{U}$, Zinserling $\mathrm{J}$ et al. The effects of different ventilator settings on pulmonary and systemic inflammatory responses during major surgery. Anaesth Analg. 2004;98:775-781.

17. Ahlers $\mathrm{O}$, Nachtigall I, Lenze $\mathrm{J}$ et al. Intraoperative thoracic epidural anaesthesia attenuates stress-induced immunosuppression in patients undergoing major abdominal surgery. Br J Anaesth. 2008;101:781-787.

18. Mokart D, Capo C, Blache JL et al. Early postoperative compensatory anti-inflammatory response syndrome is associated with septic complications after major surgical trauma in patients with cancer. Br J Surg. 2002;89:1450-1456.

19. Del Vecchio M, Bajetta E, Canova S et al. Interleukin-12: biological properties and clinical application. Clin Cancer Res. 2007;13:4677-4685.

20. Kato A, Graul-Layman A, Edwards MJ, Lentsch AB. Promotion of hepatic ischemia/reperfusion injury by IL-12 is independent of STAT4. Transplantation. 2002;73:1142-1145.

21. Yang Z, Szabo C. Production of interleukin-12 is under the control of endogenous interleukin-10 in myocardial ischemia-reperfusion. Shock. 2001;15:77-79.

22. Hutchings A, Wu J, Asiedu $\mathrm{C}$ et al. The immune decision toward allograft tolerance in non-human primates requires early inhibition of innate immunity and induction of immune regulation. Transpl Immunol. 2003;11:335-344.

23. De Perrot $\mathrm{M}$, Sekine $\mathrm{Y}$, Fisher $\mathrm{S}$ et al. Interleukin-8 release during early reperfusion predicts graft function in human lung transplantation. Am J Respir Crit Care Med. 2002;165: 211-215.

24. Bulger EM, Cuschieri J, Warner K, Maier RV. Hypertonic resuscitation modulates the inflammatory response in patients with traumatic hemorrhagic shock. Ann Surg. 2007;245:635-641. 